\title{
Association between Comorbidities and Preserved Ratio Impaired Spirometry: Using the Korean National Health and Nutrition Examination Survey IV-VI
}

\author{
Joohae Kim ${ }^{a}$ b Chang-Hoon Lee ${ }^{c}$ Ha Youn Lee ${ }^{d}$ Ho Kimª \\ aDepartment of Public Health Science, Graduate School of Public Health, Seoul National University, Seoul, Republic \\ of Korea; bivision of Pulmonary and Critical Care Medicine, Department of Internal Medicine, National Medical \\ Center, Seoul, Republic of Korea; 'Division of Pulmonary and Critical Care Medicine, Department of Internal \\ Medicine, Seoul National University Hospital, Seoul, Republic of Korea; ${ }^{\mathrm{d} D i v i s i o n}$ of Pulmonary and Critical Care \\ Medicine, Department of Internal Medicine, Serim Hospital, Incheon, Republic of Korea; 'Institute for Sustainable \\ Development, Seoul National University, Seoul, Republic of Korea
}

\section{Keywords}

Preserved ratio impaired spirometry . Prevalence .

Comorbidities - Obstructive lung disease

\begin{abstract}
Background: Preserved ratio impaired spirometry (PRISm) patients have more frequent respiratory symptoms and an increased risk of mortality. However, studies on comorbidities in these patients are lacking. Objectives: We investigated the association between PRISm and comorbidities using the Korea National Health and Nutrition Examination Survey (KNHANES). Method: This cross-sectional study included participants aged $\geq 50$ years from the KNHANES (2007-2015). Participants who did not undergo spirometry or performed inadequately were excluded. We classified participants into 3 groups according to spirometry: PRISm (forced expiratory volume in one second [FEV1] /forced vital capacity [FVC] $\geq$ 0.7 and FEV $1<80 \%$ ), chronic obstructive pulmonary disease (COPD) (FEV1/ FVC <0.7), and normal. Multivariate logistic regression analyses were used to evaluate the risk of comor-
\end{abstract}

bidities in the PRISm group compared to that in the normal group. Result: The study included 17,515 participants: 12,777 (73.0\%), 1,563 (8.9\%), and 3,175 (18.1\%) in normal, PRISm, and COPD groups, respectively. After adjustment for known risk factors of each disease, hypertension (adjusted odds ratio [95\% confidence interval]; 1.31 [1.14-1.50]), diabetes (1.51 [1.29-1.78]), hypercholesterolemia (1.20 [1.04$1.37])$, obesity (1.31 [1.15-1.48]), ischemic heart disease (1.58 [1.13-2.22]), chronic renal disease (2.31 [1.09-4.88]), and thyroid disease (1.41 [1.09-1.83]) risks were significantly higher in the PRISm group than in the normal group. The average number of comorbidities was 2.45 in the PRISm group, which was higher than that in the normal (2.1) and COPD (2.03) groups $(p<0.05)$. Conclusion: The number of comorbidities was significantly higher in the PRISm group than in others. Hypertension, diabetes, obesity, ischemic heart disease, chronic renal disease, and thyroid disease were associated with PRISm after adjustment for risk factors.

(c) 2021 The Author(s). Published by S. Karger AG, Basel
C 2021 The Author(s).

Published by S. Karger AG, Basel

This is an Open Access article licensed under the Creative Commons Attribution-NonCommercial-4.0 International License (CC BY-NC) (http://www.karger.com/Services/OpenAccessLicense), applicable to the online version of the article only. Usage and distribution for commercial purposes requires written permission.
Correspondence to:

Ho Kim, hokim@snu.ac.kr 


\section{Introduction}

Preserved ratio impaired spirometry (PRISm) is characterized by decreased forced expiratory volume in one second (FEV1 $<80 \%$ predicted) without airflow limitation (FEV1/ forced vital capacity [FVC] $\geq 0.7$ ), and is also called "GOLD unclassified" or "Restrictive spirometry." The risk of chronic obstructive pulmonary disease (COPD) development was increased in PRISm patients, despite no initial observation of airway obstruction $[1,2]$. Not only for COPD, several studies showed the association between PRISm and extrapulmonary disease, like obesity, hypertension, diabetes, and metabolic syndrome [3-5]. Restrictive spirometry was associated with diabetes in patients without known pulmonary disease [6] and was also significantly more frequent in patients with chronic renal disease [7]. Risk of developing end-stage renal disease was higher in restrictive spirometry in a long-term follow-up study [8]. In addition to increased risk of comorbidities, increased mortality risk was associated in patients with restrictive disease, after adjustment of risk factors, including age, sex, and body mass index (BMI) [2, $9,10]$.

Most studies mentioned above investigated restrictive lung disease. Though PRISm and restrictive spirometry were considered to have similar characteristics, or to be the same disease for patients with overlap, diagnostic criteria of the 2 groups are different in actuality. A few studies about PRISm have been conducted, and none of them investigated various comorbidities. We intended to investigate clinical characteristics and comorbidities of PRISm in a population-based national survey. In addition, we investigated the difference of health-care utilization by comparing the number of admitted patients in the past year.

\section{Materials and Methods}

\section{Study Population}

The Korea National Health and Nutrition Examination Survey (KNHANES) is a cross-sectional national surveillance system that consists of a health interview, a health examination, and a nutrition survey. The survey has been conducted every 3 years from 1998 to 2005 and has changed to an annual rotation sample survey since 2007. The sampling was done by stratified multistage cluster sampling design. The spirometry was performed in adults aged 19 years and over in 2007-2009, 40 years and over in 2010-2012, and between 40 and 80 years old in 2013-2015.

We used the data from 2007 to 2015 (the KNHANES IV VI) that contained information about hospital admissions occurring within 1 year prior to the survey. We included participants older than 50 years old, considering prevalence of comorbidities according to age $[11,12]$. Participants who did not undergo spirometry or performed inappropriate spirometry were excluded. This study used data from the KNHANES, which was approved by the Institutional Review Board Committee of the Korea Centers for Disease Control (IRB No.1401-047-547). All participants signed a written informed consent.

\section{Definition}

Patients whose FEV1 /FVC was $>0.7$ were classified as PRISm group when FEV 1 was $<80 \%$ of predicted percent, otherwise they were classified as normal group. If FEV1/ FVC was $<0.7$, participants were classified as obstructive group. Economic state in the original questionnaire was classified into 4 groups, namely low, mid-low, high-low, and high. Among them, we combined mid-low and high-low into "mid" group. Ever smoker was defined as a person with a smoking history of $>5$ pack-years. Alcohol consumption was classified into 3 groups. Heavy drinkers were defined as drinking $>21$ glasses for men and 14 glasses for women. Drinking less than those criteria was defined as moderate.

Comorbidities were defined in 2 ways. First, presence of comorbidity was identified by the questionnaire. Second, prevalence was investigated based on the definition by physical examination and laboratory data. Hypertension was defined as systolic blood pressure $\geq 140 \mathrm{~mm} \mathrm{Hg}$ or diastolic blood pressure $\geq 90 \mathrm{~mm} \mathrm{Hg}$. Diabetes was defined as fasting blood sugar $\geq 126 \mathrm{mg} / \mathrm{dL}$ or hemoglobin A $1 c \geq 6.5 \%$. Hypercholesterolemia was defined as total cholesterol $\geq 240 \mathrm{mg} / \mathrm{dL}$, and obesity as $\mathrm{BMI} \geq 25$. Anemia was defined as hemoglobin values $<13 \mathrm{~g} / \mathrm{dL}$ in men and $<12 \mathrm{~g} / \mathrm{dL}$ in women. We calculated the number of comorbidities by summing up the total number of affected disease for each participant. For cancers, only 1 point was counted if there were multiple primary cancers in different organs. The cause of hospitalization was identified according to a disease code based on the Korean Standard Classification of Diseases.

\section{Statistical Analysis}

We compared baseline characteristics among 3 groups by using $\chi^{2}$ test for categorical variable and analysis of variance for continuous variable. The prevalence of comorbidities was also analyzed by using $\chi^{2}$ test, and risk of comorbidity was analyzed by univariate and multivariate logistic regression. Multivariate logistic regression was adjusted for each risk factor as follows: (1) age, sex-adjusted: obesity, chronic renal disease, chronic hepatitis, anemia, thyroid disease, rheumatic disease, and depression; (2) age, sex, and BMI-adjusted: hypertension, diabetes, hypercholesterolemia, liver cirrhosis, osteoarthritis, and osteoporosis; (3) age, sex, BMI, hypertension, diabetes, hypercholesterolemia, smoking, and alcohol-adjusted: stroke and ischemic heart disease. Statistical analyses were conducted by the SAS 9.4 (SAS Institute Inc., Cary, NC, USA). Statistical significance ( $p$ value) was $<0.05$.

\section{Results}

\section{Baseline Characteristics}

There were 73,353 who participated in the KNHANES from 2007 to 2015 . In total, 17,515 participants were ana- 
Fig. 1. Flowchart. KNHANES, Korea National Health and Nutrition Examination Survey; PRISm, preserved ratio impaired spirometry; FEV1, forced expiratory volume in one second; FVC, forced vital capacity.

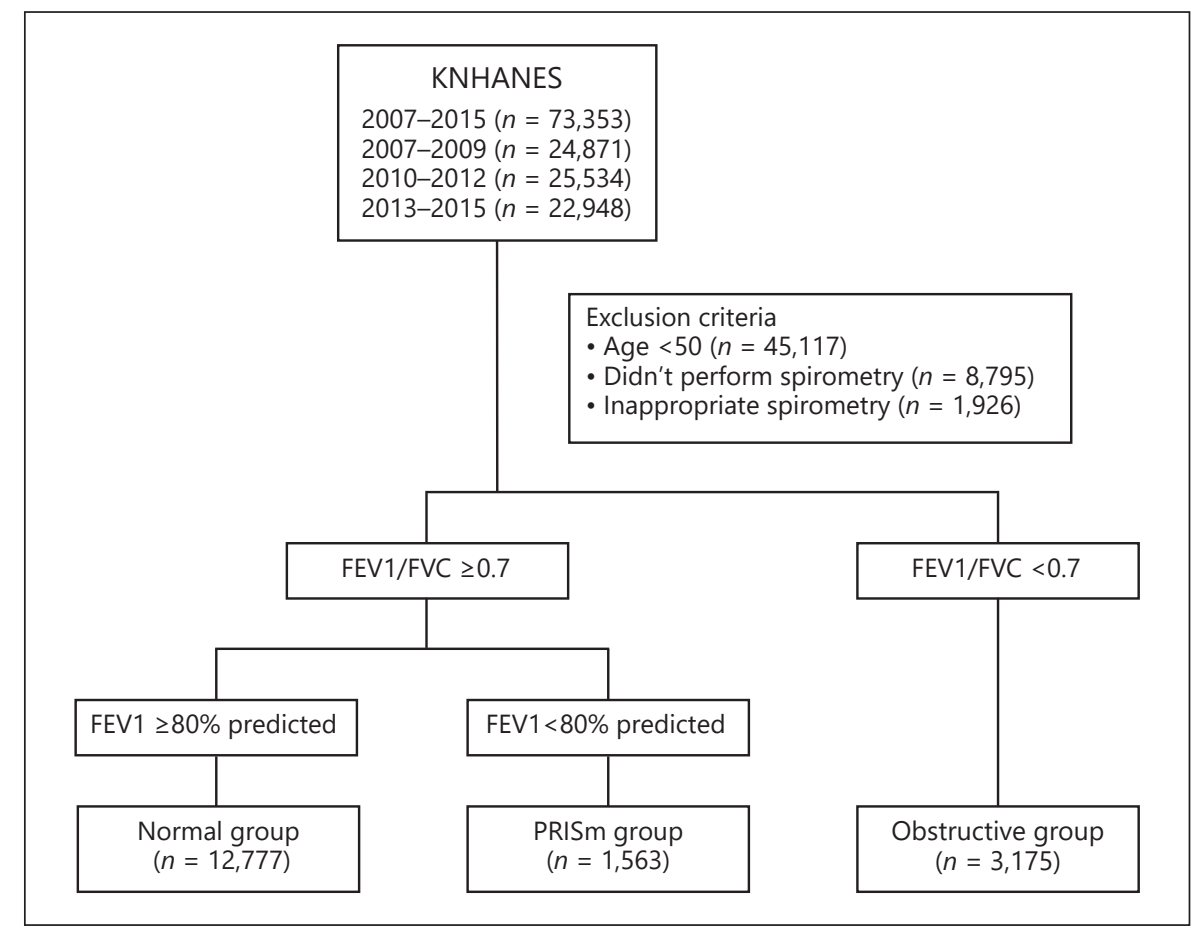

lyzed after excluding 45,117 participants who were younger than 50 years old, 8,795 participants who did not perform spirometry, and 1,926 participants who performed inadequate spirometry (Fig. 1) Among them, $73.0 \%$ (12,777 participants) was in normal spirometry group, $18.1 \%$ (3,175 participants) was in the obstructive group, and $8.9 \%$ (1,563 participants) was in PRISm group.

Average age was lowest in the PRISm group at 59.0 years old, followed by the normal group at 59.6 years old, and the obstructive group, highest at 64.7 years old (Table 1). There were significant differences for age and sex among the 3 groups, with the prevalence of PRISm being higher in women $(9.3 \%)$ than in men $(8.4 \%)$, and the obstructive group having a higher proportion of men (73.8\%). For socioeconomic status, income and education statuses were similar between the PRISm and the normal group. The proportion of "Low economic status" was significantly higher in the obstructive group than the other 2 groups, and education level was lowest in the obstructive group. The average BMI was highest in the PRISm group (24.7), followed by the normal group (24.4), and the obstructive group (23.6).

Average value of FEV1 $(2.13 \pm 0.01)$, FEV 1 predicted $\%$ $(74.0 \pm 0.2 \%)$, FVC $(2.80 \pm 0.02)$, and FVC predicted $\%$ $(74.8 \pm 0.2 \%)$ were lowest in the PRISm group and were significantly lower than the normal group. For the ob- structive group, only the FVC value was higher than in the normal group while the rest of the values were significantly lower. FEV 1/FVC ratio was 0.63 in the obstructive group, 0.76 in the PRISm group, and 0.79 in the normal group.

Proportions of ever smoker and current smoker were highest in the obstructive group (68.9 and 31.9\%, respectively). The PRISm group had less ever smokers (42.9\%) and current smokers $(23.1 \%)$ than the obstructive group, but had the highest average pack-year. Alcohol consumption, which was the proportion of participants who drank alcohol, and heavy drinkers, are also highest in the obstructive group.

\section{Prevalence of Comorbidities}

The prevalence of comorbidities is shown in Table 2. In the PRISm group, hypertension was most common (51.5\%), followed by obesity (45.4\%), hypercholesterolemia (31.2\%), and diabetes (24.0\%). Seventy-five participants $(4.9 \%)$ had ischemic heart disease and 59 participants $(3.3 \%)$ had a history of stroke. Compared to the normal group, hypertension, diabetes, hypercholesterolemia, obesity, stroke, ischemic heart disease, chronic renal disease, thyroid disease, and osteoarthritis were more prevalent in the PRISm group. Hypertension, diabetes, stroke, and ischemic heart disease were more common in 
Table 1. Baseline characteristics

\begin{tabular}{|c|c|c|c|c|c|}
\hline & $\begin{array}{l}\text { Total } \\
(N=17,515)\end{array}$ & $\begin{array}{l}\text { Normal group } \\
(N=12,777)\end{array}$ & $\begin{array}{l}\text { PRISm group } \\
(N=1,563)\end{array}$ & $\begin{array}{l}\text { Obstructive group } \\
(N=3,175)\end{array}$ & $p$ value \\
\hline Age, mean & $60.4 \pm 0.1$ & $59.6 \pm 0.1^{*, \dagger}$ & $59.0 \pm 0.2^{\ddagger}$ & $64.7 \pm 0.2$ & $<0.001$ \\
\hline \multicolumn{6}{|l|}{ Sex, $n(\%)$} \\
\hline Male & $7,495(47.5)$ & $4,572(41.5)^{*, \dagger}$ & $627(46.6)^{\ddagger}$ & $2,296(73.8)$ & \multirow{2}{*}{$<0.001$} \\
\hline Female & $10,020(52.5)$ & $8,205(58.5)^{*, \dagger}$ & $936(53.4)^{\ddagger}$ & $879(26.2)$ & \\
\hline Mid & $8,769(50.3)$ & $6,396(50.4)^{\dagger}$ & $768(50.2)$ & $1,605(50.2)$ & \multirow[t]{2}{*}{0.009} \\
\hline Low & $3,947(22.7)$ & $2,820(22.1)^{\dagger}$ & $352(22.5)$ & $775(25.4)$ & \\
\hline \multicolumn{6}{|l|}{ Education, $n(\%)$} \\
\hline$\leq$ Elementary & $7,368(38.6)$ & $5,292(37.6)^{\dagger}$ & $614(34.5)^{\ddagger}$ & $1,462(45.1)$ & \multirow{3}{*}{$<0.001$} \\
\hline Middle & $3,144(19.5)$ & $2,288(19.4)^{\dagger}$ & $302(20.2)^{\ddagger}$ & $554(19.4)$ & \\
\hline High & $4,246(27.3)$ & $3,106(27.5)^{\dagger}$ & $421(30.8)^{\ddagger}$ & $719(24.5)$ & \\
\hline FEV1, L & $2.57 \pm 0.01$ & $2.69 \pm 0.01^{*, \dagger}$ & $2.13 \pm 0.01$ & $2.27 \pm 0.02$ & $<0.001$ \\
\hline FEV1, predicted $\%$ & $91.6 \pm 0.1$ & $96.9 \pm 0.1^{*, \dagger}$ & $74.0 \pm 0.2^{\ddagger}$ & $77.9 \pm 0.3$ & $<0.001$ \\
\hline FVC, L & $3.39 \pm 0.01$ & $3.42 \pm 0.01^{*, \dagger}$ & $2.80 \pm 0.02^{\ddagger}$ & $3.58 \pm 0.02$ & $<0.001$ \\
\hline FVC, predicted \% & $91.9 \pm 0.1$ & $94.5 \pm 0.1^{*, \dagger}$ & $74.8 \pm 0.2^{\ddagger}$ & $90.2 \pm 0.3$ & $<0.001$ \\
\hline FEV1/FVC & $0.761 \pm 0.001$ & $0.790 \pm 0.001^{*, \dagger}$ & $0.764 \pm 0.001^{\ddagger}$ & $0.633 \pm 0.001$ & $<0.001$ \\
\hline \multicolumn{6}{|l|}{ Smoking, $n(\%)$} \\
\hline Nonsmoker & $10,564(57.5)$ & $8,585(63.7)^{*, \dagger}$ & $957(57.1)^{\ddagger}$ & $1,022(31.1)$ & \multirow{3}{*}{$<0.001$} \\
\hline Ex-smoker & $3,947(23.8)$ & $2,448(21.2)^{*, \dagger}$ & $301(19.8)^{\ddagger}$ & $1,198(37.0)$ & \\
\hline Current smoker & $2,664(18.7)$ & $1,501(15.1)^{*, \dagger}$ & $275(23.1)^{\ddagger}$ & $888(31.9)$ & \\
\hline Pack-year, mean & $27.7 \pm 1.6$ & $25.7 \pm 1.8$ & $37.3 \pm 10.9$ & $29.3 \pm 1.2$ & 0.188 \\
\hline \multicolumn{6}{|l|}{ Alcohol, $n(\%)$} \\
\hline
\end{tabular}

PRISm, preserved ratio impaired spirometry; FEV1, forced expiratory volume in one second; FVC, forced vital capacity; BMI, body mass index. * Normal group versus PRISm group $p<0.05$. ${ }^{\dagger}$ Normal group versus obstructive group $p<0.05$. ${ }^{\ddagger}$ PRISm group versus obstructive group $p<0.05$.

the obstructive group than in the normal group. Average number of comorbidities was 2.45 in the PRISm group, which had significantly more comorbidities than the normal (2.1) or obstructive (2.03) group.

The risk of comorbidity of PRISm and obstructive group was compared with that of normal spirometry group (Table 3). Without adjustment, risk of hypertension, diabetes, stroke, and ischemic heart disease significantly increased in both the PRISm and obstructive group. In addition, obesity, chronic kidney disease, and thyroid disease also showed higher risk in the PRISm group than the normal group. After adjustment for risk factors, there was no significant difference in stroke in the PRISm group compared to the normal group, but it was still significant in other diseases: hypertension (aOR 1.31 [95\% confidence interval (CI) 1.14-1.50]), diabetes (aOR 1.51 [95\% CI 1.29-1.78]), hypercholesterolemia (aOR 1.20 [95\% CI 1.04-1.37]), obesity (aOR 1.31 [95\% CI 1.15-1.48]), ischemic heart disease (aOR 1.58 [95\% CI 1.13-2.22]), chronic kidney disease (aOR 2.31 [95\% CI 1.09-4.88]), and thyroid disease (aOR 1.41 [95\% CI 1.091.83]). The subgroup analysis stratified by BMI showed that the risk of hypertension (aOR 1.27 [95\% CI 1.061.52]), diabetes (aOR 1.59 [95\% CI 1.26-2.00]) and chronic kidney disease (aOR 4.48 [95\% CI 1.99-10.12]) was increased even in the PRISm group without obesity $(\mathrm{BMI}<25 \mathrm{~kg} / \mathrm{m} 2)$ (see online suppl. Table 1; see www. karger.com/doi/10.1159/000517599 for all online suppl. 
Table 2. Prevalence of comorbidities

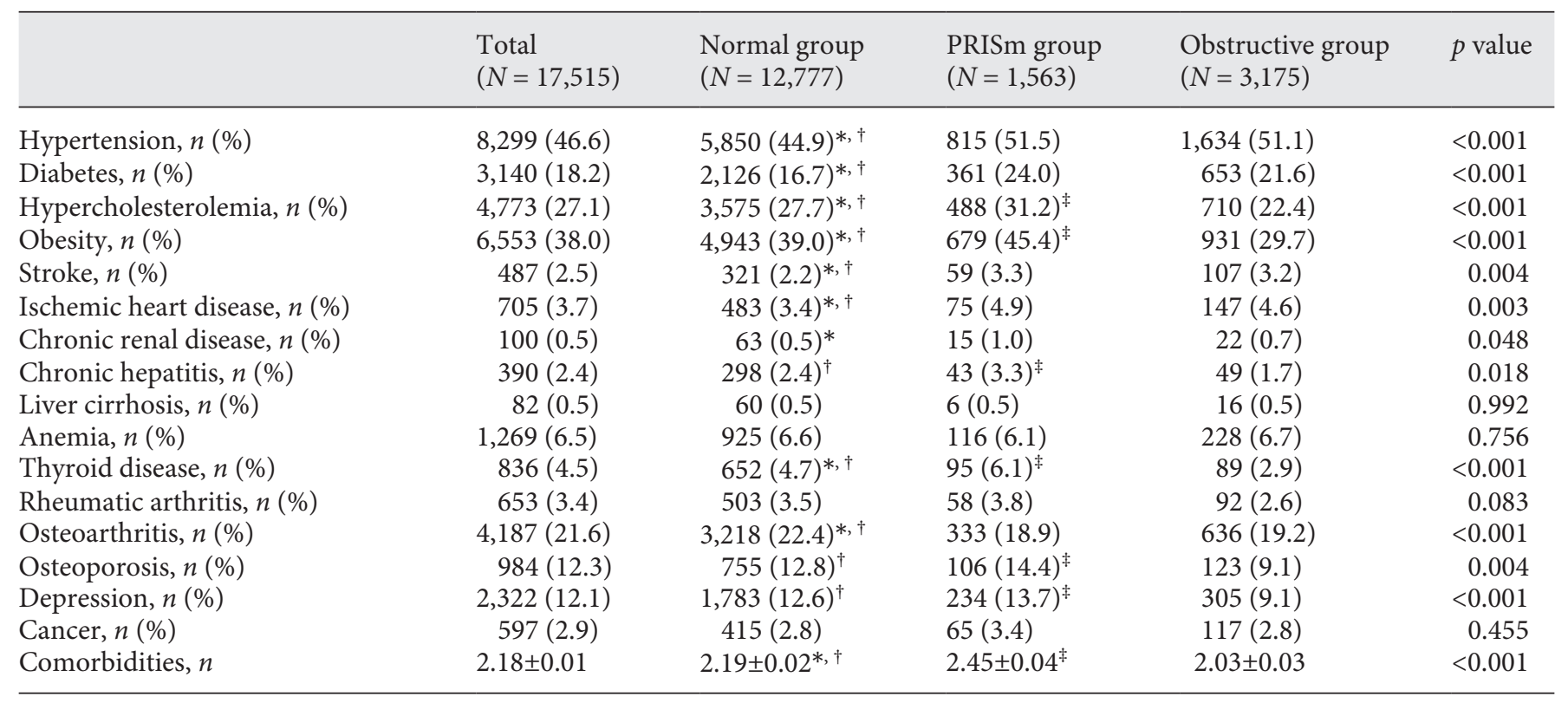

PRISm, preserved ratio impaired spirometry. *Normal group versus PRISm group $p<0.05$. Normal group versus obstructive group $p<0.05$. ${ }^{\ddagger}$ PRISm group versus obstructive group $p<0.05$.

Table 3. Risk of comorbidities in PRISm and obstructive group compared to normal group

\begin{tabular}{|c|c|c|c|c|}
\hline & \multicolumn{2}{|c|}{ Unadjusted OR (95\% CI) } & \multicolumn{2}{|c|}{ Adjusted OR $(95 \% \mathrm{CI})^{\star}$} \\
\hline Hypertension & $1.30(1.15-1.48)$ & $1.28(1.17-1.41)$ & $1.31(1.14-1.50)$ & $0.96(0.86-1.07)$ \\
\hline Diabetes & $1.58(1.35-1.84)$ & $1.37(1.22-1.55)$ & $1.51(1.29-1.78)$ & $1.05(0.92-1.19)$ \\
\hline Hypercholesterolemia & $1.18(1.03-1.35)$ & $0.75(0.67-0.85)$ & $1.20(1.04-1.37)$ & $0.90(0.80-1.02)$ \\
\hline Ischemic heart disease & $1.50(1.11-2.03)$ & $1.38(1.09-1.74)$ & $1.58(1.13-2.22)$ & $1.02(0.78-1.34)$ \\
\hline Chronic renal disease & $2.27(1.07-4.83)$ & $1.55(0.86-2.78)$ & $2.31(1.09-4.88)$ & $1.64(0.89-3.01)$ \\
\hline Chronic hepatitis & $1.38(0.94-2.02)$ & $0.68(0.47-0.98)$ & $1.32(0.90-1.94)$ & $0.62(0.42-0.91)$ \\
\hline Liver cirrhosis & $0.99(0.38-2.59)$ & $0.96(0.51-1.80)$ & $0.91(0.35-2.35)$ & $0.73(0.37-1.44)$ \\
\hline Anemia & $0.92(0.73-1.16)$ & $1.02(0.85-1.22)$ & $0.99(0.78-1.25)$ & $0.95(0.78-1.14)$ \\
\hline Thyroid disease & $1.31(1.01-1.70)$ & $0.59(0.45-0.78)$ & $1.41(1.09-1.83)$ & $1.06(0.79-1.40)$ \\
\hline Cancer & $1.23(0.88-1.71)$ & $1.01(0.78-1.31)$ & $1.30(0.93-1.81)$ & $0.96(0.74-1.26)$ \\
\hline
\end{tabular}

PRISm, preserved ratio impaired spirometry; FEV1, forced expiratory volume in one second; FVC, forced vital capacity; OR, odds ratio; $\mathrm{CI}$, confidence interval. 
Table 4. The number of hospital admissions and length of hospital stay in the previous one year

\begin{tabular}{|c|c|c|c|c|c|}
\hline & $\begin{array}{l}\text { Total } \\
(N=17,515)\end{array}$ & $\begin{array}{l}\text { Normal group } \\
(N=12,777)\end{array}$ & $\begin{array}{l}\text { PRISm group } \\
(N=1,563)\end{array}$ & $\begin{array}{l}\text { Obstructive group } \\
(N=3,175)\end{array}$ & $p$ value \\
\hline \multicolumn{6}{|l|}{ Total admission } \\
\hline Patients, $n(\%)$ & $1,944(11.3)$ & $1,338(10.7)^{\dagger}$ & $196(11.9)$ & $410(13.4)$ & 0.002 \\
\hline \multicolumn{6}{|l|}{ Respiratory } \\
\hline Patients, $n(\%)$ & $120(0.6)$ & $65(0.5)^{*, \dagger}$ & $16(1.0)$ & $39(1.2)$ & $<0.001$ \\
\hline Hospital days & $10.4 \pm 1.4$ & $9.7 \pm 2.2$ & $13.6 \pm 3.7$ & $10.2 \pm 1.3$ & 0.648 \\
\hline \multicolumn{6}{|l|}{ Cardiology } \\
\hline Patients, $n(\%)$ & $184(1.0)$ & $113(0.8)^{*, \dagger}$ & $25(1.3)$ & $46(1.3)$ & 0.013 \\
\hline Hospital days & $7.6 \pm 0.9$ & $5.9 \pm 0.7$ & $10.7 \pm 4.9$ & $10.3 \pm 1.9$ & 0.064 \\
\hline \multicolumn{6}{|l|}{ Gastrointestinal } \\
\hline Patients, $n(\%)$ & $187(1.1)$ & $125(1.0)$ & $20(1.1)$ & $42(1.3)$ & 0.247 \\
\hline Hospital days & $12.9 \pm 4.5$ & $15.5 \pm 6.5$ & $6.9 \pm 2.1$ & $7.7 \pm 1.2$ & 0.448 \\
\hline \multicolumn{6}{|l|}{ Infection } \\
\hline Patients, $n(\%)$ & $57(0.3)$ & $44(0.3)$ & $3(0.2)$ & $10(0.3)$ & 0.324 \\
\hline Hospital days & $23.1 \pm 10.7$ & $10.0 \pm 1.5$ & $38.3 \pm 16.3$ & $63.3 \pm 42.1$ & 0.100 \\
\hline \multicolumn{6}{|l|}{ Cancer } \\
\hline Patients, $n(\%)$ & $152(0.8)$ & $102(0.8)$ & $11(0.4)^{\ddagger}$ & $39(1.1)$ & 0.029 \\
\hline Hospital days & $14.9 \pm 2.5$ & $13.7 \pm 1.6$ & $9.9 \pm 2.4$ & $19.7 \pm 9.3$ & 0.315 \\
\hline \multicolumn{6}{|l|}{ Endocrinology } \\
\hline Patients, $n(\%)$ & $34(0.2)$ & $24(0.2)$ & $3(0.2)$ & $7(0.2)$ & 0.839 \\
\hline Hospital days & $18.3 \pm 5.1$ & $17.4 \pm 5.1$ & $15.2 \pm 5.6$ & $22.4 \pm 16.4$ & 0.901 \\
\hline \multicolumn{6}{|l|}{ Neurology } \\
\hline Patients, $n(\%)$ & $27(0.2)$ & $19(0.2)$ & $3(0.1)$ & $5(0.1)$ & 0.671 \\
\hline Hospital days & $10.4 \pm 2.7$ & $6.9 \pm 1.0$ & $8.2 \pm 1.9$ & $32.0 \pm 14.5$ & 0.145 \\
\hline \multicolumn{6}{|l|}{ Neuropsychiatry } \\
\hline Patients, $n(\%)$ & $9(0.04)$ & $7(0.04)$ & 0 & $2(0.05)$ & - \\
\hline Hospital days & $17.0 \pm 6.6$ & $19.5 \pm 8.1$ & - & $7.9 \pm 1.1$ & 0.153 \\
\hline \multicolumn{6}{|l|}{ Genitourinary } \\
\hline Patients, $n(\%)$ & $75(0.4)$ & $62(0.5)$ & $3(0.2)$ & $10(0.4)$ & 0.236 \\
\hline Hospital days & $8.2 \pm 1.9$ & $8.0 \pm 2.2$ & $7.4 \pm 4.5$ & $9.6 \pm 2.0$ & 0.832 \\
\hline \multicolumn{6}{|l|}{ Musculoskeletal } \\
\hline Patients, $n(\%)$ & $384(2.1)$ & $275(2.1)$ & 35 (1.9) & $74(2.0)$ & 0.785 \\
\hline Hospital days & $18.0 \pm 1.4$ & $16.5 \pm 1.0$ & $29.5 \pm 13.5$ & $19.2 \pm 2.4$ & 0.374 \\
\hline
\end{tabular}

PRISm, preserved ratio impaired spirometry; FEV1, forced expiratory volume in one second; FVC, forced vital capacity. ${ }^{*}$ Normal group versus PRISm group $p<0.05 .{ }^{\dagger}$ Normal group versus obstructive group $p<0.05$. ${ }^{\ddagger}$ PRISm group versus obstructive group $p<0.05$.

material). In the obstructive group, only hypercholesterolemia (aOR 0.88 [95\% CI 0.80-0.98]), obesity (aOR 0.64 [95\% CI 0.58-0.71]), and chronic liver disease (aOR 0.62 [95\% CI 0.42-0.91]) were significantly lower than in the normal group after adjusting risk factors.

\section{Hospitalization}

1,944 participants (11.3\%) had been admitted to the hospital within 1 year prior to the survey (Table 4). Among the 3 groups, there were significant differences in the proportion of patients who had been admitted for respiratory disease, cardiologic disease, and malignan$c y$, respectively $(p<0.001)$. The proportion of patients admitted for respiratory disease was highest in the obstructive group (1.2\%), followed by the PRISm group $(1.0 \%)$, and the normal group (0.5\%). Admission for cardiological disease was similar in both the PRISm and obstructive group (1.3\%) and was significantly lower in the normal group $(0.8 \%)$. However, cancer-related admission was lowest in the PRISm group $(0.4 \%)$, followed by the normal group (0.8\%), and the obstructive group (1.1\%). Otherwise, there was no significant difference among the 3 groups. Average hospital stay length also showed no difference among the 3 groups for all causative diseases including respiratory, cardiology, and cancer. 


\section{Discussion}

In this study, the prevalence of comorbidities in PRISm was investigated and compared with that in the normal lung function group using the KNHANES. In total, 17,515 participants, who were older than 50 years and had appropriately performed spirometry, were analyzed; the PRISm group comprised 1,563 participants (8.9\%), which was similar to those in the previous studies showing a prevalence of 6-13\% [2, 13-15], with a higher prevalence in women $[2,16,17]$. Although the proportion of ever smokers and current smokers was higher in the PRISm group in this study, further investigation is needed to determine the association between smoking and PRISm. In the COPD gene cohort, PRISm had a higher proportion of current smokers with higher average pack-years [4]. Only a few studies have included never smokers, and 1 recent study reported that smoking was inversely associated with PRISm [18].

We investigated the prevalence of comorbidities among the 3 groups and found that PRISm was significantly associated with hypertension, diabetes, hypercholesterolemia, obesity, ischemic heart disease, chronic renal disease, and thyroid disease, even after adjusting for each comorbidity risk factors. Although BMI is a common risk factor for these diseases and was highest in the PRISm group, the association was still significant after adjustment, and subgroup analysis showed an increased risk for hypertension, diabetes, and CKD in the nonobese participants $\left(\mathrm{BMI}<25 \mathrm{~kg} / \mathrm{m}^{2}\right)$.

The pathophysiologic mechanisms remain unclear, but previous research has shown a relationship between reduced FEV1 and arterial stiffness [19]. High blood pressure was also associated with lower FEV1 and FVC values, and this was significant in the very high blood pressure group $(\geq 180 / 110 \mathrm{~mm} \mathrm{Hg}$ ) [20]. In addition, the risk of other metabolic diseases increased in patients with PRISm. Obesity is a well-known risk factor for restrictive lung disease and PRISm, as excessive body fat can restrict mechanical function and reduce lung volume [21]. However, as mentioned previously, the association between comorbidities and PRISm cannot be explained by obesity alone, as there are other factors that have been suggested to lead to comorbidities and lung function impairment, such as oxidative stress, hypoxia, and chronic inflammation [22]. Oxidative stress caused by diabetes can cause lung parenchymal damage, which leads to fibrosis and structural changes [23]. Diabetic patients showed an increase in the alveolar epithelium or endothelial capillary thickness, with decreased degradation of glycated con- nective tissue [24], and a higher incidence of pulmonary fibrosis [25]. Hypoxemia also induces renal damage and inflammation, and chronic renal disease shares common risk factors with PRISm, such as diabetes and hypertension. In a study that investigated the incidence of endstage renal disease, the cumulative incidence was highest in restrictive pattern spirometry (9.8\%), and obstructive pattern spirometry showed the same cumulative incidence as that in the low normal pattern group (3.7\%) [8]. In addition, the risk of microalbuminuria was also related to restrictive pattern spirometry, but not to the obstructive pattern [26].

The proportion of patients with thyroid disease was higher in the PRISm group. Hyperthyroidism and, more evidently, hypothyroidism can reduce respiratory drive or muscle function [27], resulting in restrictive spirometry. Additionally, hypothyroidism was found to be more frequent in patients with idiopathic pulmonary fibrosis, which involves restrictive pulmonary impairment [28]. However, since the specific type of disease was not investigated in this survey, further investigation is needed to assess whether hyper- or hypothyroidism is associated with PRISm.

Furthermore, we found that admissions for respiratory or cardiological diseases were significantly higher in the PRISm group. In a previous study, PRISm patients visited the hospital more frequently for both outpatient and inpatient care, including emergency room cases [29]. These results may be related to the previously reported poor prognosis of PRISm. Although the frequency of cancer admissions showed a significant difference, its purpose was often for diagnosis or staging rather than treatment. Therefore, it was difficult to determine whether these differences were clinically significant.

The prevalence of hypertension, diabetes, stroke, and ischemic heart disease was significantly higher in the COPD group, but the risk of these comorbidities did not increase after adjusting for the risk factors for each disease. Only the OR for obesity was significantly lower in the COPD group than that in the normal group. A large cross-sectional study conducted in France showed a similar result, in which the restrictive group was significantly associated with metabolic syndromes and its components, namely, dyslipidemia, impaired fasting glucose, hypertension, and abdominal obesity [30]. However, obstructive lung disease showed no significant association, except for abdominal obesity [30].

This is the first study to investigate the relationship between overall comorbidities and PRISm with adjustment for risk factors for each comorbidity. In addition, 
contrary to most previous studies that only included ever smokers, we included never smokers, allowing the results of this study to be generalized more easily. Despite these findings, this study had several limitations. First, we conducted a cross-sectional study; thus, we could not evaluate causal relationships and were only able to analyze the association between PRISm and comorbidities. Second, previous medical history was investigated through a questionnaire in the survey, in which recall bias could have occurred. We defined the prevalence of comorbidities using physical examination and laboratory data based on the diagnostic criteria of each disease to minimize this bias. Third, there were no parameters to assess the severity of respiratory symptoms.

\section{Conclusion}

In this study, participants with PRISm had a higher prevalence of hypertension, diabetes, obesity, stroke, ischemic heart disease, chronic renal disease, thyroid disease, and osteoarthritis than that in the normal spirometry group. After adjusting for each risk factor, hypertension, diabetes, obesity, ischemic heart disease, chronic renal disease, and thyroid disease were significantly associated with PRISm. Therefore, possible comorbidities should be considered in patients with PRISm, and regular follow-up is required. In addition, we recommend further research to enhance our findings.

\section{Statement of Ethics}

The KNHANES, which was used in this study, was approved by the Institutional Review Board Committee of the Korea Centers for Disease Control (IRB No.1401-047-547). All participants signed a written informed consent.

\section{Conflict of Interest Statement}

The authors have no conflicts of interest to declare.

\section{Funding Sources}

KNHANES has been financially supported by the Health Promotion Fund of Korea with administrative support from the Ministry of Health and Welfare. This research was also supported by Policy Research Program funded by National Medical Center, Research Institute (Grant No. NMC2020-MS-06).

\section{Author Contributions}

J.K. contributed statistical analysis, data interpretation, drafting of manuscript, and final approval of the manuscript. C.-H.L. contributed study design and final approval of the manuscript. H.Y.L. contributed critical revision and final approval of the manuscript. H.K. contributed study design, statistical analysis, and final approval of the manuscript.

\section{References}

1 Sood A, Petersen H, Qualls C, Meek PM, Vazquez-Guillamet R, Celli BR, et al. Spirometric variability in smokers: transitions in COPD diagnosis in a five-year longitudinal study. Respir Res. 2016 Nov 10;17(1):147.

2 Wan ES, Fortis S, Regan EA, Hokanson J, Han MK, Casaburi R, et al. Longitudinal phenotypes and mortality in preserved ratio impaired spirometry in the COPDGene study. Am J Respir Crit Care Med. 2018 Dec 1; 198(11):1397-405.

3 Wan ES, Hokanson JE, Murphy JR, Regan EA, Make BJ, Lynch DA, et al. Clinical and radiographic predictors of GOLD-unclassified smokers in the COPDGene study. Am J Respir Crit Care Med. 2011 Jul 1;184(1):5763.

4 Wan ES, Castaldi PJ, Cho MH, Hokanson JE, Regan EA, Make BJ, et al. Epidemiology, genetics, and subtyping of preserved ratio impaired spirometry (PRISm) in COPDGene. Respir Res. 2014 Aug 6;15:89.
5 Wijnant SRA, De Roos E, Kavousi M, Stricker BH, Terzikhan N, Lahousse L, et al. Trajectory and mortality of preserved ratio impaired spirometry: the Rotterdam Study. Eur Respir J. 2019 Oct 10.

6 van den Borst B, Gosker HR, Zeegers MP, Schols AM. Pulmonary function in diabetes: a metaanalysis. Chest. 2010 Aug;138(2):393406.

7 Navaneethan SD, Mandayam S, Arrigain S, Rahman M, Winkelmayer WC, Schold JD. Obstructive and restrictive lung function measures and CKD: national health and nutrition examination survey (NHANES) 20072012. Am J Kidney Dis. 2016 Sep;68(3):41421.

8 Sumida K, Kwak L, Grams ME, Yamagata K, Punjabi NM, Kovesdy CP, et al. Lung function and incident kidney disease: the Atherosclerosis Risk in Communities (ARIC) Study. Am J Kidney Dis. 2017 Nov;70(5):675-85.
9 Guerra S, Sherrill DL, Venker C, Ceccato CM Halonen M, Martinez FD. Morbidity and mortality associated with the restrictive spirometric pattern: a longitudinal study. Thorax. 2010 Jun;65(6):499-504.

10 Vaz Fragoso CA, Gill TM, McAvay G, Yaggi HK, Van Ness PH, Concato J. Respiratory impairment and mortality in older persons: a novel spirometric approach. J Investig Med. 2011 Oct;59(7):1089-95.

11 Piccirillo JF, Vlahiotis A, Barrett LB, Flood KL, Spitznagel EL, Steyerberg EW. The changing prevalence of comorbidity across the age spectrum. Crit Rev Oncol Hematol. 2008 Aug;67(2):124-32.

12 Veenendaal $M$, Westerik JAM, van den Bemt L, Kocks JWH, Bischoff EW, Schermer TR. Age- and sex-specific prevalence of chronic comorbidity in adult patients with asthma: a real-life study. NPJ Prim Care Respir Med. 2019 Apr 29;29(1):14. 
13 Mannino DM, Ford ES, Redd SC. Obstructive and restrictive lung disease and functional limitation: data from the Third National Health and Nutrition Examination. J Intern Med. 2003 Dec;254(6):540-7.

14 Soriano JB, Miravitlles M, García-Río F, Muñoz L, Sánchez G, Sobradillo V, et al. Spirometrically-defined restrictive ventilatory defect: population variability and individual determinants. Prim Care Respir J. 2012 Jun 21(2):187-93.

15 Backman H, Eriksson B, Hedman L, Stridsman C, Jansson SA, Sovijärvi A, et al. Restrictive spirometric pattern in the general adult population: methods of defining the condition and consequences on prevalence. Respir Med. 2016 Nov; 120:116-23.

16 Kurth L, Hnizdo E. Change in prevalence of restrictive lung impairment in the U.S. population and associated risk factors: the National Health and Nutrition Examination Survey (NHANES) 1988-1994 and 2007-2010. Multidiscip Respir Med. 2015;10(1):7.

17 Siddharthan T, Grigsby M, Miele CH, Bernabe-Ortiz A, Miranda JJ, Gilman RH, et al. Prevalence and risk factors of restrictive spirometry in a cohort of Peruvian adults. Int J Tuberc Lung Dis. 2017 Sep 1;21(9):1062-8.

18 Schwartz A, Arnold N, Skinner B, Simmering J, Eberlein M, Comellas AP, et al. Preserved ratio impaired spirometry in a spirometry database. Respir Care. 2021 Jan;66(1):58-65.
19 Jankowich MD, Taveira T, Wu WC. Decreased lung function is associated with increased arterial stiffness as measured by peripheral pulse pressure: data from NHANES III. Am J Hypertens. 2010;23(6):614-9.

20 Schnabel E, Nowak D, Brasche S, Wichmann HE, Heinrich J. Association between lung function, hypertension and blood pressure medication. Respir Med. 2011 May;105(5): 727-33.

21 Mafort TT, Rufino R, Costa CH, Lopes AJ. Obesity: systemic and pulmonary complications, biochemical abnormalities, and impairment of lung function. Multidiscip Respir Med. 2016;11:28.

22 Pinto Pereira LM, Seemungal TA, Teelucksingh S, Nayak BS. Restrictive pulmonary deficit is associated with inflammation in suboptimally controlled obese diabetics. J Thorac Dis. 2013;5(3):289-97.

23 Hsia CC, Raskin P. Lung function changes related to diabetes mellitus. Diabetes Technol Ther. 2007 Jun;9(Suppl 1):S73-82.

24 Yang J, Xue Q, Miao L, Cai L. Pulmonary fibrosis: a possible diabetic complication. Diabetes Metab Res Rev. 2011 May;27(4):311-7.
25 Ehrlich SF, Quesenberry CPJr, Van Den Eeden SK, Shan J, Ferrara A. Patients diagnosed with diabetes are at increased risk for asthma, chronic obstructive pulmonary disease, pulmonary fibrosis, and pneumonia but not lung cancer. Diabetes Care. 2010 Jan; 33(1):55-60.

26 Yoon JH, Won JU, Ahn YS, Roh J. Poor lung function has inverse relationship with microalbuminuria, an early surrogate marker of kidney damage and atherosclerosis: the 5th Korea National Health and Nutrition Examination Survey. PloS One. 2014;9(4):e94125e25.

27 Ali ER. Assessment of functional lung impairment in patients with thyroid disorders. Egypt J Bronchol. 2016 Sep 1;10(3):337-47.

28 Oldham JM, Kumar D, Lee C, Patel SB, Takahashi-Manns S, Demchuk C, et al. Thyroid disease is prevalent and predicts survival in patients with idiopathic pulmonary fibrosis. Chest. 2015;148(3):692-700

29 Park HJ, Byun MK, Rhee CK, Kim K, Kim HJ, Yoo KH. Significant predictors of medically diagnosed chronic obstructive pulmonary disease in patients with preserved ratio impaired spirometry: a 3-year cohort study. Respir Res. 2018 Sep 24;19(1):185.

30 Leone N, Courbon D, Thomas F, Bean K, Jégo $B$, Leynaert B, et al. Lung function impairment and metabolic syndrome: the critical role of abdominal obesity. Am J Respir Crit Care Med. 2009 Mar 15;179(6):509-16. 